\title{
Atherogenic Index of Plasma (AIP): A Marker of Risk for Ischemic Stroke
}

\author{
Jadda Lou P. Sajo, MD ${ }^{\mathrm{a}}$, Anna Marie Sage-Nolido, MD, FPNA ${ }^{\mathrm{b} *}$ \\ email: jaddasajo@gmail.com \\ ${ }^{a b}$ Makati Medical Center, Makati City, 1229, Philippines
}

\begin{abstract}
Background

Atherogenic Index of Plasma (AIP) has been shown as a good surrogate marker for early atherogenesis in patients with cardiovascular disease. Atherosclerosis has been implicated in the pathogenesis of cerebrovascular disease and its leading comorbidities such as hypertension, diabetes, obesity and smoking. This study aims to determine the possibility of Atherogenic Index of Plasma as a marker of risk for ischemic stroke, to determine its relationship to hypertension, diabetes, obesity and smoking as well as its relationship to carotid artery stenosis and intracranial stenosis.

Methods and Results

This is a cross-sectional analytic study done in a tertiary hospital. A total of 64 patients (45 males and 19 females) diagnosed with acute or subacute ischemic stroke were included in the study. History of hypertension, diabetes and dyslipidemia were recorded. Lipid profile (Total cholesterol, Triglycerides, High Density Lipoprotein and Low Density Lipoprotein), Body Mass Index, presence and severity of intracranial stenosis on MRA and presence of carotid artery stenosis and carotid intima-media thickness were recorded. Atherogenic Index of Plasma was positively correlated with total cholesterol $[\mathrm{r}(62)=.300, \mathrm{p}=.016]$, triglycerides $[\mathrm{r}(62)=.760, \mathrm{p}=.000]$, smoking $[\mathrm{p}=.05]$, and is negatively correlated with HDL $[\mathrm{r}(62)=-.572, \mathrm{p}=.000]$ and age $[\mathrm{r}(62)=-.311, \mathrm{p}=.012]$. AIP did not show any correlation with hypertension, diabetes, intracranial stenosis, carotid artery stenosis and intima-media thickness.

\section{Conclusion}

Atherogenic index of plasma showed positive association with other lipid parameters consistent with findings in literature. No significant association was seen with other comorbidities which was probably influenced by the selected population of the study. Still, AIP shows a potential as a surrogate marker for early atherogenesis which is an important mechanism for ischemic strokes.
\end{abstract}

Published by IJRP.ORG. Selection and/or peer-review under responsibility of International Journal of Research Publications (IJRP.ORG) 


\section{Introduction}

Stroke is the second leading of cause of death in the Philippines after cardiac diseases and is also second in the leading cause of death worldwide following ischemic heart disease [1]. Despite early interventions and exercising preventing measures it still remains a disease burden in the country. Studies are now directed in prevention of stroke in association with the major causes such as carotid stenosis, intracranial stenosis and determining early markers for stroke together with the traditional risk factors such as hypertension, diabetes and obesity.

Carotid artery atherosclerosis has been thought to contribute to approximately $15 \%$ of strokes. Carotid artery stenosis is a narrowing or constriction of any part of the carotid arteries usually caused by atherosclerosis. The NASCET has defined the degree of stenosis as $0-40 \%$ low-degree stenosis, $50-60 \%$ moderate stenosis and $>70 \%$ as high degree or hemodynamically relevant stenosis [2]. In a study by Flaherty, et al, the most important cause of large vessel stroke was extracranial internal carotid stenosis and was associated $8 \%$ of all ischemic strokes while intracranial atherosclerosis and extracranial ICA occlusion was associated with $3.5 \%$ of the cases of ischemic strokes [3] In contrast to the different stroke types, intracranial atherosclerotic stenosis of a major intracranial artery is associated with high risk of recurrent stroke. It is associated with up to $30-50 \%$ of strokes in Asian population. This is associated with hypertension, smoking, diabetes mellitus and dyslipidemia [10].

Studies have shown that decreased high density lipoprotein, high levels of total cholesterol, triglyceride and low density lipoprotein are contributory to atherosclerosis which is a pathophysiological process in stroke [4]. Intracranial stenosis, too, is a common cause of ischemic stroke and contributes to a high risk recurrent stroke. Among Asian population, about 30-50\% is brought about by intracranial stenosis [11]. According to the Warfarin Aspirin Symptomatic Intracranial Disease (WASID) trial, both cholesterol levels and elevated blood pressure are the most important modifiable risk factor for vascular events include ischemic stroke [12].

Atherogenic index of plasma (AIP) is considered as a potential high sensitivity index of atherosclerosis for assessment of cardiovascular risk factors in predicting acute coronary events [4]. It is based on 2 parameters: serum triglyceride, and serum HDL-c. The ratio of these factors reflects the metabolism of lipoproteins which can be a useful tool in predicting plasma atherogenicity [5]. AIP is defined as logarithm [log] of the ratio of plasma concentration of TG to HDL-C [4] and reflects the association of atherogenic and protective lipoproteins [6]. Studies have suggested that AIP value below 0.11 is associated with low risk for cardiovascular disease, 0.11-0.21 with intermediate risk and values above 0.21 associated with increased risk [7].

Several studies have shown the relationship of Atherogenic Index of Plasma to cardiovascular disease and its risk factors including obesity, hypertension and diabetes mellitus. A case control study done in Chinese Han population revealed AIP as a significant independent predictor for coronary artery disease. AIP was a parameter that was strongly associated with coronary artery disease with its value noted to be higher in Coronary Artery Disease group than in control group [4]. In a study by Zhu et al., higher AIP level showed strong association with obesity [8]. Edwards, et al., discussed a strong correlation of elevated AIP with mortality risk in older US adults as compared to individual cholesterol factors [9]. However, little is known of its relationship to acute stroke and its role as an early risk marker for ischemic stroke, carotid atherosclerosis and intracranial stenosis. Sujatha, et al., discussed the positive relationship of lipid indices in stroke patients. 
The use of different lipid indices by calculating parameters from the lipid profile showed a significant relationship in stroke patients as compared to inconsistent results using lipid parameters. The study showed that lipid indices may show a true risk between stroke and lipid abnormality as compared to individual lipid markers alone [10]. Future researches are still directed to find cost effective alternatives to other costly diagnostic tests to determine the risk for stroke.

Vast research has been done to explore areas where one can prevent the occurrence of stroke. Identifying risk factors associated with stroke is essential as stroke is one of the leading causes of morbidity and mortality in the Philippines. This research would like to explore the possibility of a newer approach to identifying early atherosclerosis with a method that is non-expensive and would only use values from routine lipid determination as sensitive surrogate marker for atherosclerosis and its association with ischemic stroke.

\section{Methodology}

This is a Cross - Sectional Analytic study. Convenience sampling method was used to achieve the sample size in this study. The sample size was computed using an online software, OpenEpi, Version 3. Based on previous studies, the percentage of Ischemic stroke patients with AIP $>0.1$ is $96.6 \%$ (Sujatha, R., \& Kavitha, S., 2017) [10]. The margin of error used is 5\%. At $95 \%$ confidence level and accounting for $20 \%$, result showed that the minimum required sample size is 64 . Continuous recruitment of subjects was done until the sample size of 64 was reached. Adult patients (aged 18 years old and above) admitted in a tertiary hospital within the period of 6 months (September 2019 - March 2020) diagnosed with acute to subacute ischemic stroke thru Magnetic Resonance Imaging with Magnetic Resonance Angiography of the intracranial vessels by a licensed Radiologist done within the institution and clinically diagnosed as an ischemic stroke by a licensed Neurologist. All patients admitted at a tertiary institution within the duration of study diagnosed with acute or subacute ischemic infarct who fit the inclusion criteria were identified. Inclusion criteria are as follows: (1) adult aged 18 years old and above; (2) radiologically and clinically diagnosed case of acute to subacute ischemic infarct by a licensed Neurologist and thru imaging studies: Magnetic Resonance Imaging of the brain and Magnetic Resonance Angiography of the Intracranial Vessels; (3) with a baseline lipid profile determination and carotid and vertebral ultrasound done on current admission. Exclusion criteria includes: (1) refused to give informed consent; (2) did not undergo Carotid and Vertebral Doppler study or baseline lipid profile determination; (3) presented with an intraparenchymal hemorrhage on radiologic imaging; (4) have an unclear diagnosis of an acute or subacute ischemic stroke.

Data collection started once the Institutional Review Board (IRB) has given the final decision that the study is approved. Informed consent was secured from either the patient or a legal representative. History and radiologic findings were reviewed. Baseline characteristics of the patient were gathered such as age, sex, body mass index (derived from height and weight), comorbidities namely Hypertension, Diabetes, Dyslipidemia or previous use of anti-dyslipidemic agents and history of smoking. Data on the patient's serum lipid profile was also collected. Atherogenic index of plasma was derived using the formula $\log 10$ (Triglycerides/HDL-C). The given value was characterized as either low risk, intermediate risk or high risk. Carotid and vertebral ultrasonography was used to assess for carotid or vertebral stenosis and intima-media thickness. Magnetic Resonance Angiography results were gathered also to assess for presence intracranial stenosis. The data was accessible through reviewing the patient's charts as well as thru the Electronic Medical Records (EMR) system. Imaging results was available thru the Picture Archiving and Communication (PACS) system. The Atherogenic Index of Plasma (AIP) was correlated to the risk factors of Ischemic stroke. AIP was correlated to the degree of atherosclerosis by evaluation upon carotid and vertebral doppler study done by a licensed radiologist and presence of intracranial stenosis evaluated by MRA by licensed radiologist. 


\section{Statistical Data/Analysis}

Participant characteristics were described by percentage, means and standard deviation (SD). The correlation between AIP and the other continuous variables was calculated by Pearson correlation analysis. Chi-Square Test of Independence was used for categorical variables (X2); Fisher's Exact T values and Likelihood Ratios were alternatively used when more than $20 \%$ of cells with expected frequencies $<5$. Statistical analysis will be performed using the Statistical Package of Social Science (SPSS). In all calculations, a $\mathrm{p}<0.05$ will be considered a significant level.

\section{Results}

The study population consisted of 64 participants diagnosed with acute or subacute ischemic strokes by MRI of the brain during their hospital admission. There were 45 males and 19 females aged 33-98 years with a mean of $59.78(\mathrm{SD} \pm 14.64)$. Table 1 shows the sociodemographic and biochemical characteristics of the studied subjects.

Table 1. Sociodemographic and Biochemical Characteristics of Subjects

\begin{tabular}{llllll}
\hline & $\mathbf{N}$ & Minimum & Maximum & Mean & Std. Deviation \\
\hline Age & 64 & 33 & 98 & 59.78 & 14.637 \\
BMI & 64 & 18.6 & 33.9 & 25.047 & 3.5135 \\
Total Cholesterol & 64 & 102.84 & 384.67 & 199.7206 & 60.44488 \\
Triglycerides & 64 & 51.33 & 1008.90 & 143.5445 & 133.30606 \\
HDL & 64 & 25.52 & 67.27 & 44.1147 & 9.93907 \\
LDL & 64 & 29.38 & 303.09 & 138.7580 & 52.82505 \\
AIP & 64 & -.43 & 1.16 & .0992 & .28526 \\
\hline
\end{tabular}

Among the 64 study participants diagnosed with ischemic stroke, 46 (71.87\%) have hypertension, 22 $(34.37 \%)$ have diabetes mellitus, and $8(12.5 \%)$ had a prior history of cerebrovascular disease, among which 7 had an ischemic stroke and 1 had a prior transient ischemic attack. Twenty-three participants (35.94\%) had a history of dyslipidemia or use of cholesterol-lowering drugs and $33(51.56 \%)$ had a history of smoking. Overall, the mean BMI was 25.05 ( $\mathrm{SD} \pm 3.51$ ), with 33 participants $(51.56 \%$ ) within the normal range and 28 $(43.75 \%)$ in the overweight range.

The mean Atherogenic Index of Plasma (AIP) was 0.09 ( $\mathrm{SD} \pm 0.288)$ with 34 patients $(53.12 \%)$ in the low risk group, 8 patients $(12.5 \%)$ in the intermediate risk group, and 22 patients $(34.38 \%)$ in the increased risk group. There was a mean total cholesterol of 199.72 ( $\mathrm{SD} \pm 60.44)$, mean triglycerides of 143.54 $(\mathrm{SD} \pm 133.31)$ with one subject with a value of 1008.9, mean HDL of $44.11(\mathrm{SD} \pm 9.94)$, and mean LDL of 138.76 ( $\mathrm{SD} \pm 52.82$ ). Among all the patients, 31 (48.44\%) had elevated total cholesterol, 18 (28.12) had elevated triglycerides, 58 (90.62\%) had low HDL, and 48 (75\%) had elevated LDL.

Pearson Correlation Analysis was used to investigate the correlation of AIP with other continuous variables. Table 2 summarizes the correlation of AIP with individual lipid markers, age and body mass index. 
AIP was positively correlated with total cholesterol $[\mathrm{r}(62)=.300, p=.016]$ and triglycerides $[\mathrm{r}(62)=.760$, $p=.000]$, and negatively correlated with HDL $[\mathrm{r}(62)=-.572, p=.000]$ and age $[\mathrm{r}(62)=-.311, p=.012]$.

Table 2. Correlation Between AIP and the Individual Lipid Markers, Age and BMI

\begin{tabular}{|c|c|c|c|c|c|c|c|c|}
\hline & & AIP & $\begin{array}{l}\text { Total } \\
\text { Cholesterol }\end{array}$ & Triglycerides & HDL & LDL & Age & BMI \\
\hline \multirow{2}{*}{ AIP } & $\begin{array}{l}\text { Pearson } \\
\text { Correlation }\end{array}$ & 1 & $.300^{*}$ & $.760^{* *}$ & $-.572^{* *}$ & .079 & $-.311^{*}$ & .188 \\
\hline & Sig. (2-tailed) & & .016 & .000 & .000 & .535 & .012 & .138 \\
\hline \multirow{2}{*}{$\begin{array}{l}\text { Total } \\
\text { Cholesterol }\end{array}$} & $\begin{array}{l}\text { Pearson } \\
\text { Correlation }\end{array}$ & $.300^{*}$ & 1 & $.501^{* *}$ & $.327^{* *}$ & $.875^{* *}$ & -.160 & -.069 \\
\hline & Sig. (2-tailed) & .016 & & .000 & .008 & .000 & .207 & .588 \\
\hline \multirow[t]{2}{*}{ Triglycerides } & $\begin{array}{l}\text { Pearson } \\
\text { Correlation }\end{array}$ & $.760^{* *}$ & $.501^{* *}$ & 1 & -.174 & .091 & -.201 & .148 \\
\hline & Sig. (2-tailed) & .000 & .000 & & .168 & .474 & .112 & .245 \\
\hline \multirow[t]{2}{*}{ HDL } & $\begin{array}{l}\text { Pearson } \\
\text { Correlation }\end{array}$ & $-.572^{* *}$ & $.327^{* *}$ & -.174 & 1 & $.285^{*}$ & $.270^{*}$ & $-.267^{*}$ \\
\hline & Sig. (2-tailed) & .000 & .008 & .168 & & .022 & .031 & .033 \\
\hline \multirow[t]{2}{*}{ LDL } & $\begin{array}{l}\text { Pearson } \\
\text { Correlation }\end{array}$ & .079 & $.875^{* *}$ & .091 & $.285^{*}$ & 1 & -.199 & -.041 \\
\hline & Sig. (2-tailed) & .535 & .000 & .474 & .022 & & .115 & .747 \\
\hline
\end{tabular}

*. Correlation is significant at the 0.05 level (2-tailed). **. Correlation is significant at the 0.01 level (2-tailed).

Out of the 64 study participants, 49 (76.56\%) demonstrated atherosclerotic vessels in MRA while 15 $(23.44 \%)$ showed normal findings. Furthermore, 37 out of the $64(57.81 \%)$ showed intracranial stenosis, with $27(29.73 \%)$ having mild stenosis and $10(27.03 \%)$ having severe intracranial stenosis. Carotid ultrasound studies showed $56(87.50 \%)$ with no significant stenosis, $3(4.69 \%)$ with moderate stenosis and only 1 $(1.56 \%)$ with severe stenosis. In addition, 37 (57.71\%) had increased intima media thickness.

Atherogenic Index of Plasma (AIP) and the individual lipid markers were correlated with risk factors for stroke and imaging findings in patients with ischemic stroke as shown in Table 3. Chi Square Test was used to test for the association of AIP with other categorical values. AIP was associated with smoking $[p=.05]$. It did not show significant association with other comorbidities such as dyslipidemia, hypertension, and diabetes mellitus as well as intracranial stenosis and carotid artery stenosis. Among the individual lipid markers, only total cholesterol was associated with the severity of intracranial stenosis [ $\mathrm{p}=.047]$ while HDL was associated with a history of dyslipidemia $[\mathrm{p}=.020]$. Triglycerides and HDL did not show significant association with risk factors and imaging findings. 
Table 3. Association Between Lipid Markers, Risk Factors and Imaging Findings

\begin{tabular}{|c|c|c|c|c|c|c|}
\hline Variable & & AIP & $\begin{array}{l}\text { Total } \\
\text { Cholesterol }\end{array}$ & Triglycerides & HDL & LDL \\
\hline \multirow{4}{*}{ Sex } & Value & 2.569 & .190 & .699 & 1.309 & .225 \\
\hline & $p$ & .277 & .663 & .414 & $.351^{\mathrm{a}}$ & $.758^{\mathrm{a}}$ \\
\hline & df & 2 & 1 & 1 & 1 & 1 \\
\hline & Value & 1.277 & 1.611 & .336 & .430 & .103 \\
\hline \multirow[t]{2}{*}{ Hypertension } & $p$ & .528 & .204 & .562 & $.667^{\mathrm{a}}$ & $.756^{\mathrm{a}}$ \\
\hline & df & 2 & 1 & 1 & 1 & 1 \\
\hline \multirow{3}{*}{ Diabetes Mellitus } & Value & .905 & .119 & 2.710 & .707 & .831 \\
\hline & $p$ & .636 & .730 & .100 & $.406^{\mathrm{a}}$ & .362 \\
\hline & df & 2 & 1 & 1 & 1 & 1 \\
\hline \multirow{3}{*}{ Dyslipidemia } & Value & 1.900 & .005 & .787 & $6.460 *$ & 1.832 \\
\hline & $p$ & .387 & .942 & .375 & $.020^{\mathrm{a}}$ & .176 \\
\hline & df & 2 & 1 & 1 & 1 & 1 \\
\hline \multirow{3}{*}{ Smoking } & Value & $5.982^{\mathrm{b} *}$ & 2.231 & .160 & .881 & 1.022 \\
\hline & $p$ & .05 & .135 & .689 & $.419^{a}$ & .312 \\
\hline & df & 2 & 1 & 1 & 1 & 1 \\
\hline \multirow{3}{*}{$\begin{array}{l}\text { Atherosclerotic } \\
\text { Vessels on MRA }\end{array}$} & Value & $.413^{\mathrm{b}}$ & .188 & .021 & .169 & .726 \\
\hline & $p$ & .813 & .665 & $1.000^{\mathrm{a}}$ & $1.000^{\mathrm{a}}$ & $.498^{\mathrm{a}}$ \\
\hline & df & 2 & 1 & 1 & 1 & 1 \\
\hline \multirow{3}{*}{$\begin{array}{l}\text { Presence of } \\
\text { Intracranial } \\
\text { Stenosis on MRA }\end{array}$} & Value & 1.386 & 1.108 & .805 & .213 & 1.730 \\
\hline & $p$ & .500 & .293 & .370 & $1.000^{\mathrm{a}}$ & .188 \\
\hline & df & 2 & 1 & 1 & 1 & 1 \\
\hline \multirow{3}{*}{$\begin{array}{l}\text { Severity of } \\
\text { Intracranial } \\
\text { Stenosis on MRA }\end{array}$} & Value & $14.199^{b}$ & $11.236^{\mathrm{b} *}$ & 8.013 & $6.727^{\mathrm{b}}$ & $8.850^{\mathrm{b}}$ \\
\hline & $p$ & .077 & .047 & .156 & .242 & .115 \\
\hline & $\mathrm{df}$ & 8 & 5 & 5 & 5 & 5 \\
\hline \multirow{3}{*}{$\begin{array}{l}\text { Degree of Stenosis } \\
\text { on Carotid Doppler } \\
\text { Ultrasound }\end{array}$} & Value & $9.490^{\mathrm{b}}$ & 1.735 & $7.296^{\mathrm{b}}$ & $2.687^{\mathrm{b}}$ & $4.422^{\mathrm{b}}$ \\
\hline & $p$ & .148 & .629 & .063 & .442 & .219 \\
\hline & df & 6 & 3 & 3 & 3 & 3 \\
\hline \multirow{3}{*}{$\begin{array}{l}\text { Intima-Media } \\
\text { Thickness on } \\
\text { Carotid Doppler } \\
\text { Ultrasound }\end{array}$} & Value & 1.025 & .947 & .112 & .166 & .534 \\
\hline & $p$ & .599 & .330 & .738 & $.691^{\mathrm{a}}$ & .465 \\
\hline & $\mathrm{df}$ & 2 & 1 & 1 & 1 & 1 \\
\hline
\end{tabular}

\section{Discussion}

Among several lipid markers, atherogenic index of plasma (AIP) is one of the recent markers that is considered to be a sensitive predictor of coronary atherosclerosis and cardiovascular risk. This ratio reflects accurately the presence of atherogenic small LDL and HDL particles. AIP has been shown to be a better predictor of myocardial infarction and atherosclerotic heart disease [13], though only a few studies have shown its correlation with cerebrovascular disease. In this study, we tested for the association between AIP and other lipid markers with the risk factors for cerebrovascular disease. Majority of the participants were 
hypertensive with prior history of diabetes, a minor portion with a history of dyslipidemia while about $51 \%$ were smokers.

This study did not show a significant correlation between AIP and hypertension, diabetes mellitus, or a previous history of dyslipidemia in known stroke patients. Moreover, other lipid markers were not significantly correlated with these comorbidities as well. This may be partly attributed to the study sample, which may contain a smaller percentage of patients with these known comorbidities relative to the larger population. On the contrary, only AIP showed a positive correlation with smoking history. Smoking has been well known to affect the process of early atherogenesis, plaque formation, and plaque stability [14]. The relationship of AIP with age, on the other hand, has produced varying findings. Our study showed a negative correlation between AIP with age and this is consistent with findings by Cai et al (2017) and Hartopo et al. (2016), which also showed an inverse relationship between AIP and age $[4,15]$. In contrast to this, a study done by Nansseu et al. (2016) showed a positive correlation between these variables [16]. This difference in results may be influenced by the selected age range of the study population. In our study, majority of those in the older age group have a low risk AIP as compared to younger adults with higher proportion with the highrisk AIP values. This may be partly to due lifestyle and diet modifications as well as management of dyslipidemia and their other comorbidities with anti-dyslipidemic agents. In this present study, AIP is positively correlated with total cholesterol and triglycerides and negatively correlated with HDL. These findings are consistent with several studies including studies by Cai et al (2017) and Niroumand et al (2015) on patients with cardiovascular disease $[4,17]$.

Carotid artery duplex ultrasound is a valuable tool for assessing vascular wall atherosclerotic changes]. Increased carotid intima-media thickness and plaque formation are sensitive indications for early stage atherosclerosis [18]. In a single center study done to determine association of AIP with symptomatic carotid artery sclerosis, AIP was the only lipid parameter independently associated with symptomatic carotid artery stenosis causing ischemic stroke [18]. Carotid intima-media thickness has been shown to be a strong marker for risk for subclinical atherosclerosis. Furthermore, in a cross sectional, case control study by Cure et al. (2017), a strong relationship was noted between carotid intima-media thickness and AIP among patients with Behcet Disease, a clinical entity involving atherosclerosis with complications as its leading cause of morbidity and mortality [20]. A study by Mahat et al, (2018) also showed a positive correlation of AIP with CIMT in prediabetic patients [13]. Digital subtraction imaging has been the gold standard in identification of carotid artery stenosis but due to its cost, risks for developing stroke and radiation exposure, this is less commonly used in our institution as a baseline assessment in stroke patient during admission. Magnetic resonance angiography has improved sensitivity in detecting stenosis with better visualization of smaller arteries on contrast enhancement [21]. However, due to the cost, only a small number of stroke patients are subjected to this diagnostic modality in our institution. In this present study, results did not show a correlation with carotid intima-media thickness nor to the degree of carotid artery stenosis. As to plaque morphology, 36 (56.25\%) and 17 (26.56\%) participants have a type V and type IV plaque morphologies, respectively. These are considered the 'stable' types of plaques. While only a small portion $1(1.56 \%)$ and $3(4.69 \%)$ have the type II and type III morphology, respectively. We have observed that all participants with a type II and III plaque morphology have a high-risk AIP value. In the study of Cicorella et al (2009), unstable carotid plaques were more common in patients with acute coronary syndrome reflecting the risk of unstable plaques in developing cardiovascular disease [22]. Current studies are now directed in identifying the role of inflammation in plaque development and rupture and research has shown that risk of stroke is as well dependent on the properties of the plaque and its stability [23]. However, no prior study has determined the relationship of AIP with carotid artery plaque stability in cerebrovascular disease.

Intracranial stenosis is one of the major causes of ischemic stroke worldwide [24]. The gold standard 
in evaluating intracranial stenosis is through catheter angiography; however, due to its invasive nature with associated risk for developing stroke, this diagnostic modality is less often used. Transcranial doppler ultrasound, magnetic resonance angiography, and CT angiography are other modalities commonly used to evaluate intracranial stenosis [25]. In this study, subjects underwent Magnetic resonance angiogram, which is the preferred modality due to cost, convenience, and safety. Upon testing for a correlation between intracranial stenosis or presence of atherosclerotic vessels with AIP, we found that these factors were not significantly correlated. Among the individual lipid markers, only total cholesterol showed a positive correlation with the degree of stenosis, whereby a higher value of total cholesterol was correlated with the more severe degree of stenosis. In the study of Wang et al (2020), individual lipid markers such as LDL, Triglycerides and Total cholesterol were not significantly associated with asymptomatic intracranial stenosis while lipid ratios such as LDL-c/HDL-c ratio and TC/HDL-c were significantly correlated [26]. Unlike our findings, a low HDL was noted to be inversely related to asymptomatic intracranial stenosis. No studies have been done to test for a direct correlation between AIP and intracranial stenosis in patients with stroke.

\section{Limitation and Recommendations}

This is a cross-sectional analytical study which limits us in establishing a causal relationship between the different variables. Our study was limited to the commonly used diagnostic modalities in our institution. Identification of carotid artery stenosis, plaque characteristics and detecting intracranial stenosis are better detected with the use of gold standard imaging modalities or a combination of different imaging tests. Studies in comparison with healthy controls are recommended to better establish AIP as an early marker for risk in cerebrovascular disease. We also recommend a longer duration of study with a larger scale participants.

\section{Conclusion}

In summary, our study showed that AIP is positively correlated with other individual lipid markers: total cholesterol, triglycerides, and smoking. It is also negatively correlated with age and HDL. The limited number of participants who were included in the study and the variable baseline characteristics and comorbidities may affect the results. Still, Atherogenic Index of Plasma shows potential as a surrogate of individual lipid markers in assessing risk for Ischemic stroke. Future studies on the value of AIP are still recommended.

\section{References}

1. Stroke Society of the Philippines. SSP Handbook of Stroke Guidelines for Prevention, Treatment, and Rehabilitation 6th edition. Philippines: Golden Pages. 2014;p. 8.

2. North American Symptomatic Carotid Endarterectomy Trial (NASCET) Collaborators. Beneficial effect of carotid endarterectomy in symptomatic patients with high-grade carotid stenosis. N Engl J Med. 1991;325: 445-453.

3. Flaherty, M, et al. Carotid Artery Stenosis as a Cause of Stroke. Neuroepidemiology. 2013;40:36-41.

4. Cai, G, et al. The atherogenic index of plasma is a strong and independent predictor for coronary artery disease in the Chinese Han population. Medicine. 2017; 96:37(e8058).

5. Wu T, Gao Y, Zheng Y, Ma Y, Xie X. Atherogenic index of plasma (AIP): a novel predictive indicator for the coronary artery disease in postmenopausal women. Lipids Health Dis. 2018;17(1):197.

6. Dobiásová, M, Urbanová, Z, and Samánek, M. Relations between particle size of HDL and LDL lipoproteins and cholesterol esterification rate. Physiological Research, 2015; 54: 159-165.

7. Dobiasova M. Atherogenic index of plasma [ $\log ($ triglycerides/HDL-cholesterol)]: theoretical and practical implications. Clin Chem. 2004;50(7):1113-5.

8. Zhu X, Yu L, Zhou H, et al. Atherogenic index of plasma is a novel and better biomarker associated with obesity: a population-based 
cross-sectional study in China. Lipids Health Dis. 2018;17(1):37. Published 2018 Mar 5. doi:10.1186/s12944-018-0686-8

9. Edwards, M, Blaha, M and Loprinzi, P. Atherogenic Index of Plasma and Triglyceride/High Density Lipoprotein Cholesterol Ratio predict Mortality Risk Better than Individual Cholesterol Risk Factors, Among an Older Adult Population. Mayo Clinic Proc. 2017: 92(4): 690-686.

10. Sujatha, R, and Kavitha, S. Atherogenic indices in stroke patients: A retrospective study. Iranian journal of neurology, 2017;16(2), 78-82.

11. Holmstedt, C, Turan, T \& Chimowitz, M. Atherosclerotic intracranial arterial stenosis: risk factors, diagnosis, and treatment. The Lancet. Neurology. 2013; 12(11), 1106-1114

12. Chaturvedi S, Turan T, Lynn M, et al. Risk factor status and vascular events in patients with symptomatic intracranial stenosis Neurology. 2007; 69:2063-68.

13. Mahat R, Singh N, Rathore V, Gupta A, \& Shah R. Relationship between Atherogenic Indices and Carotid Intima-Media Thickness in Prediabetes: A Cross-Sectional Study from Central India. Med Sci (Basel). 2018; 6(3):55.

14. Messner B, Bernhard D. Smoking and cardiovascular disease: mechanisms of endothelial dysfunction and early atherogenesis. Arterioscler Thromb Vasc Biol. 2014; 34(3):509-15

15. Hartopo A, Arso, I, Setianto B. Low plasma atherogenic index associated with poor prognosis in hospitalized patients with acute myocardial infarction. Acta Med Indones. 2016; 48:106-13.

16. Nansseu J, Moor V, Nouaga M. et al. Atherogenic index of plasma and risk of cardiovascular disease among Cameroonian postmenopausal women. Lipids Health Dis. 2016; 15:49.

17. Niroumand S, Khajedaluee M, Khadem-Rezaiyan, M, et al. Atherogenic index of Plasma (AIP): a marker of cardiovascular disease. Med J Islam Repub Iran 2015;29:240.

18. Murray C, Nahar T, Kalashyan H, Becher H, Nanda N. Ultrasound assessment of carotid arteries: Current concepts, methodologies, diagnostic criteria, and technological advancements. Echocardiography. 2018 Dec;35(12):2079-2091

19. Garg, R, Knox, N., Prasad, S, Zinzuwadia, S, \& Rech, M. The Atherogenic Index of Plasma is Independently Associated with Symptomatic Carotid Artery Stenosis. Journal of Stroke and Cerebrovascular Diseases. 2020; 29(12), 105351

20. Cure E, Icli A, Ugur Uslu A, Aydoğan Baykara R, Sakiz D, Ozucan M, Yavuz F, Arslan S, Cumhur Cure M, Kucuk A. Atherogenic index of plasma may be strong predictor of subclinical atherosclerosis in patients with Behçet disease. Z Rheumatol. 2017 Apr;76(3):259-266. English.

21. Saxena, A, Ng, E, \& Lim, S. Imaging modalities to diagnose carotid artery stenosis: progress and prospect. Biomedical engineering online. $2019 ; 18(1), 66$.

22. Cicorella N, Zanolla L, Franceschini L, Cacici G, De Cristan B, Arieti M, Vassanelli C. Usefulness of ultrasonographic markers of carotid atherosclerosis (intima-media thickness, unstable carotid plaques and severe carotid stenosis) for predicting presence and extent of coronary artery disease. J Cardiovasc Med (Hagerstown). 2009; Dec;10(12):906-12.

23. Skagen, K, Skjelland, M, Zamani, M, \& Russell, D. Unstable carotid artery plaque: new insights and controversies in diagnostics and treatment. Croatian medical journal. 2016; 57(4), 311-320

24. Yaghi S, Prabhakaran S, Khatri P, Liebeskind DS. Intracranial atherosclerotic disease.Stroke. 2019; 50:1286-1293.

25. Banerjee C, Chimowitz M. Stroke caused by atherosclerosis of the major intracranial arteries. Circ Res. 2017; 120:502-513

26. Wang S, Wang X, Zhao Y, Ji X, Sang S, Shao S, Xiang Y, Wang G, Lv M, Xue F, Du Y, Qiu C, Sun Q. Characterizing lipid profiles associated with asymptomatic intracranial arterial stenosis in rural-dwelling adults: A population-based study. J Clin Lipidol. 2020 May-Jun;14(3):371-380. doi: 10.1016/j.jacl.2020.04.005. Epub 2020 Apr 19. PMID: 32389549. 